\title{
A Comparison between Hybrid and Concomitant Regimens for Helicobacter Pylori Eradication: A Randomized Clinical Trial
}

\author{
Saman Alhooei ${ }^{1}$, Hafez Tirgar Fakheri ${ }^{2 *}$, Vahid Hosseini ${ }^{3}$, Iradj Maleki ${ }^{4}$, Tarang Taghvaei ${ }^{5}$, \\ Seyed Mohammad Valizadeh ${ }^{6}$, Zohreh Bari ${ }^{7}$
}

1. Assistant Professor, Department of Gastroenterology, Golestan University of Medical Sciences, Gorgan, Iran

2. Professor, Department of Gastroenterology, Gut and Liver Research Center, Mazandaran University of Medical Sciences, Sari, Iran

3. Associate Professor, Department of Gastroenterology, Gut and Liver Research Center, Mazandaran University of Medical Sciences, Sari, Iran

4. Associate Professor, Department of Gastroenterology, Gut and Liver Research Center, Mazandaran University of Medical Sciences, Sari, Iran

5. Associate Professor, Department of Gastroenterology, Gut and Liver Research Center, Mazandaran University of Medical Sciences, Sari, Iran

6. Assistant Professor, Department of Gastroenterology, Gut and Liver Research Center, Mazandaran University of Medical Sciences, Sari, Iran

7. Fellow of Gastroenterology, Gut and Liver Research Center, Mazandaran University of Medical Sciences, Sari, Iran

\section{* Corresponding Author:}

Hafez Tirgar Fakheri, MD

Department of Gastroenterology, Gut and Liver Research Center,

Mazandaran University of Medical Sciences, Sari, Iran

Tel: + 981133377176

Fax: + 981133363754

Email: hafezfakheri@gmail.com

Received: 30 Jan. 2016

Accepted: 18 Apr. 2016

\section{ABSTRACT}

\section{BACKGROUND}

Helicobacter pylori (H. pylori) is one of the most common bacterial infections worldwide. We designed a study to compare the efficacy of 14-day hybrid regimen with 10-day concomitant therapy for $H$. pylori eradication in Iran.

\section{METHODS}

252 patients with naïve $H$. pylori infection were randomly divided to receive either hybrid regimen (pantoprazole $40 \mathrm{mg}$, and amoxicillin $1 \mathrm{gr}$ twice daily for 14 days, accompanied by clarithromycin $500 \mathrm{mg}$, and metronidazole $500 \mathrm{mg}$, twice daily just during the last 7 days) or concomitant regimen (pantoprazole $40 \mathrm{mg}$, amoxicillin 1 gr, clarithromycin $500 \mathrm{mg}$, and metronidazole $500 \mathrm{mg}$, all twice daily for 10 days). 8 weeks after therapy, ${ }^{14} \mathrm{C}$ - urease breath test was performed to confirm eradication.

\section{RESULTS}

According to intention to treat analysis, the eradication rates were $87.3 \%(95 \% \mathrm{CI}$ : 81.4-93.1) and $80.9 \%$ (95\% CI: 74-87.8) in hybrid and concomitant groups, respectively $(p=0.38)$. Per-protocol eradication rates were $89.3 \%$ (95\% CI: 83.8-94.7) and $83.1 \%$ (95\% CI: $76.3-89.8)$, respectively $(p=0.19)$. The rates of severe side effects were not statistically different between the two groups ( $4 \%$ vs. $8.7 \%)$.

\section{CONCLUSION}

14-day hybrid therapy can be considered as a nearly acceptable regimen with few severe side effects in Iran. However, it seems that the efficacy of this therapy is decreasing as the resistance rates to antibiotics are increasing. We suggest further studies to assess the efficacy of a more prolonged concomitant therapy for $\mathrm{H}$. pylori eradication in Iran.

Please cite this paper as:

Alhooei S, Tirgar Fakheri H, Hosseini V, Maleki I, Taghvaei T, Valizadeh SM, Bari Z. A Comparison between Hybrid and Concomitant Regimens for Helicobacter Pylori Eradication: A Randomized Clinical Trial. Middle East J Dig Dis 2016:219-225. DOI :10.15171/ mejdd.2016.24

\section{INTRODUCTION}

Helicobacter pylori (H. pylori) is one of the most common bacterial infections worldwide, affecting almost half of the world's population. ${ }^{1,2}$ The prevalence of $H$. pylori infection is estimated to be about $70 \%$ in the developing countries and about $30-40 \%$ in developed countries. ${ }^{3}$ The infection is associated with peptic ulcer disease, gastric adenocarcinoma, and lymphoma. ${ }^{2}$

Since 1982, many investigations have been performed to introduce a regimen with ideal $H$. pylori eradication rate. Clarithromycin-containing triple 
therapy had been the gold standard $H$. pylori eradication regimen during the previous years. But the increasing resistance to antibiotics, especially to clarithromycin, has lowered the efficacy of the mentioned therapy to less than $80 \%$ in many countries. ${ }^{4}$ Therefore, many investigators have attempted to introduce regimens with higher efficacy for $H$. pylori eradication.

Hybrid regimen is a novel $H$. pylori eradication strategy consisted of a proton pump inhibitor (PPI) plus amoxicillin during the first half of therapy, followed by a concomitant administration of a PPI plus amoxicillin, clarithromycin, and metronidazole or tinidazole. The regimen can be given in 10- or 14-day protocols..$^{5}$ This strategy has shown ideal eradication rates in recent studies. $^{6-9}$

Concomitant therapy is another novel regimen consisted of concomitant administration of a PPI plus amoxicillin, clarithromycin, and metronidazole for 10 to 14 days. Several studies have shown more than $90 \%$ per-protocol $H$. pylori eradication rates by concomitant therapy. ${ }^{10-12}$

Studies comparing concomitant regimen with hybrid therapy mostly have achieved acceptable $H$. pylori eradication rates by both regimens. These studies have mostly compared regimens with the same duration of therapy. However, in the concomitant therapy, a higher number of tablets are administered when compared with hybrid therapy with the same duration of treatment. This can lead to more frequent adverse effects by the concomitant therapy and a higher cost.

Up to now, no study has evaluated the efficacy of concomitant regimen for $H$. pylori eradication in Iran. Iran is a country with high clarithromycin resistance rate. Studies performed in countries with the same pattern of claritromycin resistance have shown acceptable H. pylori eradication rates by both 10-day and 14-day concomitant regimens. ${ }^{13-15}$ Furthermore, treatment duration of at least 7 days is recommended for the success of concomitant therapy. ${ }^{16,17}$ Therefore, we designed a study to compare the efficacy of 10-day concomitant therapy with 14-day hybrid regimen for $H$. pylori eradication in Iran. Although these two regimens have different durations, they have almost the same number of tablets (120 vs. 126, respectively).

\section{MATERIALS AND METHODS}

252 patients with peptic ulcer disease or erosive gastro/duodenopathy entered the study. They all had naïve H. pylori infection, confirmed by antral biopsy (Giemsa staining) and rapid urease test. They were randomly divided into 2 groups to receive either hybrid regimen (pantoprazole $40 \mathrm{mg}$ and amoxicillin $1 \mathrm{gr}$ twice daily for 14 days, accompanied by clarithromycin $500 \mathrm{mg}$ and metronidazole $500 \mathrm{mg}$ twice daily just during the last 7 days) or concomitant regimen (pantoprazole $40 \mathrm{mg}$, amoxicillin $1 \mathrm{gr}$, clarithromycin $500 \mathrm{mg}$, and metronidazole $500 \mathrm{mg}$, all twice daily for 10 days).

The protocol was approved by the Ethics Committee of Mazandaran University of Medical Sciences and also, written informed consents were obtained from all patients.

The patients were advised to take all the prescribed drugs and were visited at the end of treatment to evaluate their compliance to treatment and side effects of therapy. They were also advised to call the physician in case of any severe adverse reaction.

Side effects of therapy were considered severe, if they abandoned daily activities, moderate if they partially interfered with daily activities, and mild if they were easily tolerable and did not interfere with daily activities.

Compliance to treatment was considered excellent if the patient took more than $90 \%$ of the medications, moderate if he/she took $70-90 \%$ of the prescribed drugs, and poor if the patient took less than $70 \%$ of medications.

Eight weeks after therapy, ${ }^{14} \mathrm{C}$ - urease breath test $(\mathrm{He}-$ liprobe Breath Card and Analyser, Kibion AB, Uppsala, Sweden) was performed to confirm $H$. pylori eradication.

\section{Statistical analysis:}

In Iran, $H$. pylori eradication rates achieved by 14-day hybrid regimen have been about $92 \%$. On the other hand, no study has evaluated the effects of concomitant therapy on $H$. pylori eradication in Iran. In other countries, the eradication rates achieved by 14-day concomitant regimen have been about $86 \%$. Therefore, we assumed to have about $9 \%$ difference between the 14-day hybrid and 10 -day concomitant regimens in their ability to eradicate H. pylori.

Considering $\alpha=0.05 \%$ and $\beta=0.2 \%$ and the equation 
Table 1: Demographic, clinical characteristics, and endoscopic findings of the patients

\begin{tabular}{lccc}
\hline Variable & 14-day Hybrid & 10-day Concomitant & p value \\
\hline Male/ Female \% & $35 / 65$ & $39 / 61$ & 0.74 \\
\hline Age (mean \pm SD) (years) & $45.3 \pm 12.9$ & $49.2 \pm 12.3$ & 0.12 \\
\hline History of GIB* (\%) & $6(4.8)$ & $10(7.9)$ & 0.43 \\
\hline History of NSAIDs use** $(\%)$ & $14(11.1)$ & $15(11.9)$ & 0.99 \\
\hline Smokers (\%) & $18(14.3)$ & $21(16.7)$ & 0.72 \\
\hline Type of ulcer & & & \\
$-\quad$ Duodenal ulcer & $77(61.1)$ & $65(51.6)$ & 0.16 \\
$-\quad$ Gastric ulcer & $21(16.7)$ & $30(23.8)$ & 0.20 \\
$\quad$ Duodenal erosion & $21(16.7)$ & $27(21.4)$ & 0.30 \\
$-\quad$ Gastric erosion & $40(31.7)$ & $55(43.7)$ & 0.08 \\
\hline Bulbar deformity & $19(15.1)$ & $21(16.7)$ & 0.86 \\
\hline${ }^{*}$ GIB: gastrointestinal bleeding \\
${ }_{*}^{*}$ NSAIDs: Non-steroidal anti-inflammatory drugs & & &
\end{tabular}

the number of patients in each arm was calculated to be

$$
n=\frac{2\left(\mathrm{z}_{1-\overline{2}}+\mathrm{z}_{1-\bar{\beta}}\right)^{2}+\overline{\mathrm{p}}(1-\overline{\mathrm{p}})}{\left(\mathrm{p}_{1}-\mathrm{p}_{2}\right)^{2}}
$$

96. But we assumed about $20 \%$ withdrawal rate, therefore, we included at least 120 patients in each arm.

Eradication rate was evaluated according to intention to treat (ITT) and per-protocol (PP) analyses. In order to calculate ITT eradication rate, all patients who entered the study were evaluated. In order to calculate PP eradication rate, only patients who completed the entire protocol with more than $90 \%$ compliance to therapy entered the analysis. Data were analyzed using IBM SPSS software for windows (version 16; SPSS Inc., Chicago, IL, USA) and Pearson chi-square test, t test, and logistic regression analysis were used as appropriate. $\mathrm{P}$ values $<0.05$ were considered as statistically significant. The statistician was blind to the assignment of patients.

\section{RESULTS}

252 patients entered the study. 126 patients were assigned to each group. The mean ages of the patients were $45.3 \pm 12.9$ years and $42.9 \pm 12.3$ years in hybrid and concomitant groups, respectively $(p=0.12)$. Other demographic characteristics were not statistically different between the two groups (table 1).

245 patients completed the study. Three patients in hybrid group and four patients in concomitant group did not perform urea breath test. They had also interrupted therapy because of severe adverse effects.

Compliance to treatment was excellent in 122 patients $(96.8 \%)$ of hybrid therapy group, while one patient $(0.8 \%)$ had moderate, and three patients $(2.4 \%)$ had poor compliance to therapy. In concomitant group, compliance to treatment was excellent in 119 patients (94.4\%), moderate in three patients $(2.4 \%)$, and poor in four patients $(3.2 \%)$. The rates of compliance to treatment did not show significant difference between the two groups $(p=0.55)$

43 patients $(34.1 \%)$ complained of side effects of therapy in hybrid group, but the adverse effects were severe only in five patients (4\%). In concomitant group, 56 patients $(44.4 \%)$ reported side effects, which were severe in 11 patients $(8.7 \%)$. However, the rates of total side effects, as well as the rates of severe side effects were not statistically different between the two groups ( $p$ $=0.12$ and $p=0.25$, respectively)(table 2 ).

According to ITT analysis, the eradication rates were $87.3 \%$ (95\% confidence interval (CI) 81.4-93.1) and 80.9\% (95\% CI: 74-87.8) in hybrid and concomitant groups, respectively ( $p=0.38$ ). PP eradication rates were $89.3 \%$ (95\% CI: 83.8-94.7) and 83.1\% (95\% CI: 76.3$89.8)$, respectively ( $p=0.19$, figure 1$)$.

\section{DISCUSSION}

According to the results of the present study, 14-day hybrid therapy could achieve $87.3 \%$ and $89.3 \%$ H. pylori 
Table 2: Adverse effects reported by the patients during treatment

\begin{tabular}{lccc}
\hline Adverse effect & 14-day Hybrid & 10-day Concomitant & p value \\
\hline Epigastric pain & $5(4)$ & $7(5.6)$ & 0.76 \\
\hline Sleepiness & $2(1.6)$ & 0 & 0.49 \\
\hline Anxiety & $1(0.8)$ & 0 & 0.99 \\
\hline Abdominal cramp & $2(1.6)$ & $1(0.8)$ & 0.99 \\
\hline Weakness & $1(0.8)$ & $6(4.8)$ & 0.12 \\
\hline Nausea \& vomiting & $10(7.9)$ & $11(8.7)$ & 0.99 \\
\hline Metallic taste & $26(20.6)$ & $28(22.2)$ & 0.87 \\
\hline Dizziness & $6(4.8)$ & $6(4.8)$ & 1.00 \\
\hline Constipation & $2(1.6)$ & $1(0.8)$ & 0.99 \\
\hline Dysuria & $1(0.8)$ & 0 & 0.99 \\
\hline Blurred vision & 0 & $1(0.8)$ & 0.99 \\
\hline Rash & 0 & $1(0.8)$ & 0.99 \\
\hline Water brash & 0 & $3(2.4)$ & 0.24 \\
\hline Pruritus & $2(1.6)$ & 0 & 0.49 \\
\hline Headache & $3(2.4)$ & $6(4.8)$ & 0.50 \\
\hline Tremor & $1(0.8)$ & 0 & 0.99 \\
\hline Glossitis & 1 & $1(0.8)$ & 1.00 \\
\hline Stomatitis & 0 & $2(1.6)$ & 0.47 \\
\hline Diarrhea & $3(2.4)$ & $5(4)$ & 0.74 \\
\hline Bloating & $1(0.8)$ & $1(0.8)$ & 1.00 \\
\hline Fever & $1(0.8)$ & 0 & 0.99 \\
\hline Anorexia & $2(1.6)$ & $18(14.6)$ & 0.17 \\
\hline Severity of side effects & & & \\
\hline Mild & $14(11.1)$ & $(21.4)$ & \\
\hline GIB: gastrointestinal bleeding & $5(4)$ & 0.25 \\
\hline
\end{tabular}

**NSAIDs: Non-steroidal anti-inflammatory drugs

eradication rates according to ITT and PP analyses, respectively. On the other hand, the eradication rates achieved by concomitant regimen were $80.9 \%$ and $83.1 \%$, respectively.

If we consider $H$. pylori infection as an infectious disease, an ideal regimen would be the one that can eradicate H. pylori in more than $95 \%$ of cases, with less than $5 \%$ severe adverse effects. ${ }^{18}$ According to Graham's classification, the efficacy of $H$. pylori eradication regimens are considered as A: excellent ( $>95 \%$ PP eradication rate), B: good (90-95\%), C: fair (85-89\%), D: poor (81-84\%), and $\mathrm{F}$ : unacceptable ( $\leq 80 \% \mathrm{PP}$ eradication rate). ${ }^{18}$ Therefore, in our study, hybrid and concomitant regimens are classified as fair (C) and poor (D) regimens, respectively. However, the eradication rate achieved by hybrid thera- py was nearly good (89.3\%).

The rate of side effects is also an important issue. In our study, the incidence rates of adverse effects of therapy were $34.1 \%$ and $44.4 \%$ in hybrid and concomitant groups, respectively. However, they were mostly mild and the rates of severe adverse effects were $4 \%$ and $8.7 \%$, respectively. Accordingly, 14-day hybrid regimen can be considered as a nearly good regimen with few severe side effects.

In Iran, only few studies have evaluated the effects of hybrid therapy on H. pylori eradication. In 2013, Sardarian and colleagues compared the effects of 14-day hybrid regimen vs. 10-day sequential therapy on 420 patients with peptic ulcer disease. The eradication rates achieved by hybrid therapy were $89.5 \%$ and $92.9 \%$ according 

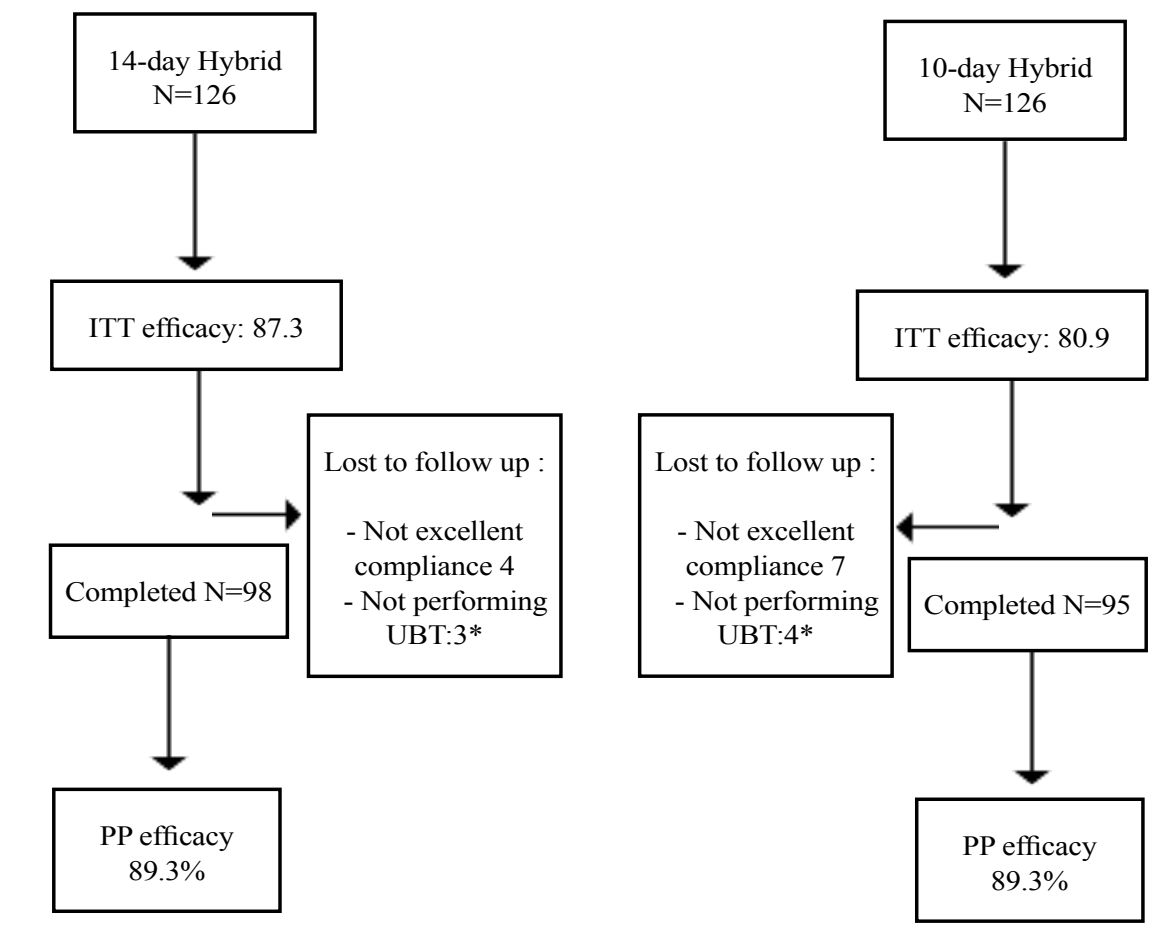

ITT: intention to treat; PP: per-protocol; UBT: Urea breath test

*These patients are among those who did not have excellent compliance to treatment.

Fig 1: Method of follow up and treatment efficacy

to ITT and PP analyses, respectively. ${ }^{19}$ Furthermore, in 2015, Metanat and co-workers compared 10-day vs. 14-day hybrid therapy on 270 patients with peptic ulcer disease in Iran. The ITT eradication rates were $77.6 \%$ and $86 \%$ for 10 -day and 14-day regimens, respectively. Also, PP eradication rates were $83.8 \%$ and $92.8 \%$, respectively. This study, highlighted the ideal efficacy of 14-day hybrid regimen for H. pylori eradication in Iran. ${ }^{20}$

Comparing the results of our study with the two previous studies performed in Iran, the lower rates of $H$. pylori eradication in our study might have been due to the increasing antibiotic resistance. In Iran, during the previous years, the rate of $H$. pylori resistance to clarithromycin has increased from $1.4 \%$ in 1997 to $14.3 \%$ in 2012. Also, the rate of resistance to metronidazole has increased from $33 \%$ to $76 \%$. $^{21,22}$

Considering concomitant therapy, no study had previously evaluated concomitant therapy for $H$. pylori eradication in Iran. Therefore, we cannot compare our results with the same studies in our country.

In other countries, hybrid and concomitant regimens have mostly achieved acceptable $H$. pylori eradication rates. In 2013, Zullo and others compared the effects of 14-day hybrid regimen with 5-day concomitant therapy on patients with non-ulcer dyspepsia in Italy. The ITT eradication rates were $80 \%$ and $85.5 \%$, respectively. Also, PP eradication rates were $85.7 \%$ and $91.6 \%$, respectively. In the mentioned study, differences in the eradication rates were not statistically significant. ${ }^{12}$

In 2014, De Francesco and colleagues compared 14day hybrid regimen with 14-day and 5-day concomitant therapies in Italy. ITT eradication rates were $82.7 \%$, $86.3 \%$, and $78.1 \%$, respectively. The differences between the eradication rates were not statistically significant between either 14-day hybrid or 14-day concomitant therapies, but the rate achieved by 5-day concomitant regimen was significantly lower than the other two groups. ${ }^{14}$

Italy is a country with high resistance rates to antibiotics against $H$. pylori, similar to our country. ${ }^{23,24}$ The two studies performed in Italy demonstrate a tendency toward decrease in $H$. pylori eradication rates by hybrid and concomitant regimens. This is similar to the results of studies performed in Iran.

In 2013, Molina-infante and co-workers compared 
14-day hybrid regimen versus 14-day concomitant regimen for H. pylori eradication in Spain. The ITT eradication rates were $90 \%$ and $91.7 \%$, respectively. Also, PP eradication rates were $92 \%$ and $96.1 \%$, respectively. Accordingly, both hybrid and concomitant regimens could achieve ideal eradication rates. ${ }^{13}$

In 2015, Molina-infante and colleagues compared the efficacy of optimized (high dose acid suppression) 14day concomitant therapy with optimized triple therapy in Spain. ${ }^{24}$ The eradication rates achieved by concomitant therapy were $90.4 \%$ and $93.8 \%$ according to ITT and PP analyses, respectively.

Although Spain is also a country with high resistance rates to antibiotics against $H$. pylori, the results of the latter two studies performed in Spain showed higher eradication rates compared with our results. These differences might have been due to differences in dual or multi-drug antibiotic resistance or the differences in patient selection criteria.

The results of studies performed in Asia are almost the same as the mentioned studies. In 2015, Heo and others compared 10-day hybrid and 10-day concomitant therapies in Korea. The eradication rates achieved by ITT analysis were $78.8 \%$ and $78.6 \%$, respectively. Also, PP eradication rates were $89.6 \%$ and $89.8 \%$, respectively. Both regimens had similar and acceptable efficacies, but hybrid regimen was better tolerated and had fewer side effects. ${ }^{25}$

In 2012, Gisbert performed a meta-analysis of the studies evaluating the effects of concomitant regimen for H. pylori eradication. 19 studies, including 2070 patients were evaluated. The ITT eradication rate was $88 \%$. Furthermore, a tendency toward higher eradication rates with 10-day versus 5-day regimens was observed. ${ }^{11}$

Also, in 2014, Lei He and co-workers performed a meta-analysis to compare sequential, concomitant, and hybrid therapies for $H$. pylori eradication. Ten randomized clinical trials consisted of 3501 patients were included. The ITT eradication rates were $86.7 \%$ and $86.6 \%$ for the concomitant and hybrid regimens, respectively. PP eradication rates were $89.8 \%$ and $92.7 \%$, respectively. This meta-analysis showed no significant difference between the two regimens in terms of $H$. pylori eradication rates or adverse effects of therapies. ${ }^{26}$

Totally, in countries with high antibiotic resistance rates, quadruple non-bismuth therapy (including concomitant, hybrid, or sequential regimens) is a first line option. ${ }^{15}$ But the results of our study and the other studies performed in other countries have mostly been borderline (near 90\%). It is thought that dual clarithromycinmetronidazole resistance is the weak point in achieving excellent eradication rates by non-bismuth quadruple therapies.

The main limitation of the present study was the unavailability of $H$. pylori culture. However, the latest study performed in our province (Mazandaran) has reported $30 \%$ resistance to clarithromycin, $73.4 \%$ resistance to metronidazole, and $6.8 \%$ to amoxicillin. ${ }^{27}$

The strong point of the present study is being the first study evaluating the effects of concomitant regimen on H. pylori eradication in Iran.

In conclusion, 14-day hybrid regimen can be considered as a nearly acceptable regimen with few severe side effects in Iran. However, it seems that the efficacy of this therapy is decreasing as the resistance rates to antibiotics are increasing. We suggest further studies to assess the efficacy of a more prolonged concomitant therapy for H. pylori eradication in Iran.

\section{ACKNOWLEDGEMENTS}

The authors acknowledge the financial support of Mazandaran University of Medical Sciences.

\section{CONFLICT OF INTEREST}

The authors declare no conflict of interest related to this work.

\section{REFERENCES}

1. Linz B, Balloux F, Moodley Y, Manica A, Liu H, Roumagnac $\mathrm{P}$, et al. An African origin for the intimate association between humans and Helicobacter pylori. Nature 2007;445:915-8. doi:10.1038/nature05562

2. Go MF. Review article: natural history and epidemiology of Helicobacter pylori infection. Aliment Pharmacol Ther 2002;16:3-15. doi: 10.1046/j.1365-2036.2002.0160s1003.x

3. Becker SI, Smalligan RD, Frame JD, Kleanthous H, Tibbitts TJ, Monath TP, et al. Risk of Helicobacter pylori infection among long-term residents in developing countries. Am J Trop Med Hyg 1999;60:267-70.

4. Tokunaga K, Tanaka A, Sugano H, Takahashi S. [The present status and problems of Helicobacter pylori firstline eradication therapy]. Nihon Rinsho 2009;67:2291-6. 
5. Hsu PI, Wu DC, Wu JY, Graham DY. Modified sequential Helicobacter pylori therapy: proton pump inhibitor and amoxicillin for 14 days with clarithromycin and metronidazole added as a quadruple (hybrid) therapy for the final 7 days. Helicobacter 2011;16:139-45. doi: 10.1111/j.1523-5378.2011.00828.x.

6. Kim SY, Lee SW, Hyun JJ, Jung SW, Koo JS, Yim HJ, et al. Comparative study of Helicobacter pylori eradication rates with 5-day quadruple "concomitant" therapy and 7-day standard triple therapy. J Clin Gastroenterol 2013;47:21-4. doi: 10.1097/MCG.0b013e3182548ad4.

7. Kongchayanun C, Vilaichone RK, Pornthisarn B, Amornsawadwattana S, Mahachai V. Pilot studies to identify the optimum duration of concomitant Helicobacter pylori eradication therapy in Thailand. Helicobacter 2012;17:282-5. doi: 10.1111/j.1523-5378.2012.00953.x.

8. O'Connor A, Molina-Infante J, Gisbert JP, O'Morain C. Treatment of Helicobacter pylori infection 2013. Helicobacter 2013;1:58-65. doi: 10.1111/hel.12075.

9. Wu JY, Liou JM, Graham DY. Evidence-based recommendations for successful Helicobacter pylori treatment. Expert Rev Gastroenterol Hepatol 2014;8:21-8. doi: 10.1586/17474124.2014.859522.

10. Wu DC, Hsu PI, Wu JY, Opekun AR, Kuo CH, Wu IC, et al. Sequential and concomitant therapy with four drugs is equally effective for eradication of $H$ pylori infection. Clin Gastroenterol Hepatol 2010;8:36-41.e1. doi: 10.1016/j.cgh.2009.09.030.

11. Gisbert JP, Calvet X. Update on non-bismuth quadruple (concomitant) therapy for eradication of Helicobacter pylori. Clin Exp Gastroenterol 2012;5:23-34. doi: 10.2147/ CEG.S25419.

12. Zullo A, Scaccianoce G, De Francesco V, Ruggiero V, D'Ambrosio P, Castorani L, et al. Concomitant, sequential, and hybrid therapy for $\mathrm{H}$. pylori eradication: a pilot study. Clin Res Hepatol Gastroenterol 2013;37:647-50. doi: 10.1016/j.clinre.2013.04.003.

13. Molina-Infante J, Romano M, Fernandez-Bermejo M, Federico A, Gravina AG, Pozzati L, et al. Optimized nonbismuth quadruple therapies cure most patients with Helicobacter pylori infection in populations with high rates of antibiotic resistance. Gastroenterology 2013;145:121-8 e1. doi: 10.1053/j.gastro.2013.03.050.

14. De Francesco V, Hassan C, Ridola L, Giorgio F, Ierardi E, Zullo A. Sequential, concomitant and hybrid first-line therapies for Helicobacter pylori eradication: a prospective randomized study. $J$ Med Microbiol 2014;63:748-52. doi: 10.1099/jmm.0.072322-0.

15. Molina-Infante J, Gisbert JP. Optimizing clarithromycincontaining therapy for Helicobacter pylori in the era of antibiotic resistance. World J Gastroenterol 2014;20:1033847. doi: 10.3748/wjg.v20.i30.10338.

16. Essa AS, Kramer JR, Graham DY, Treiber G. Meta-analysis: four-drug, three-antibiotic, non-bismuth-containing "concomitant therapy" versus triple therapy for Helicobacter pylori eradication. Helicobacter 2009;14:109-18. doi: 10.1111/j.1523-5378.2009.00671.x.

17. Papastergiou V, Georgopoulos SD, Karatapanis S. Treatment of Helicobacter pylori infection: Past, present and future. World J Gastrointest Pathophysiol 2014;5:392-9. doi: 10.4291/wjgp.v5.i4.392.

18. Graham DY, Lu H, Yamaoka Y. A report card to grade Helicobacter pylori therapy. Helicobacter 2007;12:275-8. doi: 10.1111/j.1523-5378.2007.00518.x

19. Sardarian H, Fakheri H, Hosseini V, Taghvaei T, Maleki I, Mokhtare M. Comparison of hybrid and sequential therapies for Helicobacter pylori eradication in Iran: a prospective randomized trial. Helicobacter 2013;18:129-34. doi: 10.1111/hel.12017.

20. Metanat HA, Valizadeh SM, Fakheri H, Maleki I, Taghvaei $\mathrm{T}$, Hosseini $\mathrm{V}$, et al. Comparison Between 10- and 14-Day Hybrid Regimens for Helicobacter pylori Eradication: A Randomized Clinical Trial. Helicobacter 2015;20:299-304. doi: 10.1111/hel.12202.

21. Milani M, Ghotaslou R, Akhi MT, Nahaei MR, Hasani A, Somi MH, et al. The status of antimicrobial resistance of Helicobacter pylori in Eastern Azerbaijan, Iran: comparative study according to demographics. J Infect Chemother 2012;18:848-52. doi: 10.1007/s10156-012-0425-4.

22. Safaralizadeh R, Siavoshi F, Malekzadeh R, Akbari MR, Derakhshan MH, Sohrabi MR, et al. Antimicrobial effectiveness of furazolidone against metronidazole-resistant strains of Helicobacter pylori. East Mediterr Health $J$ 2006;12:286-93.

23. Saracino IM, Zullo A, Holton J, Castelli V, Fiorini G, Zaccaro $\mathrm{C}$, et al. High prevalence of primary antibiotic resistance in Helicobacter pylori isolates in Italy. $J$ Gastrointestin Liver Dis 2012;21:363-5.

24. Agudo S, Alarcon T, Cibrelus L, Urruzuno P, Martinez MJ, Lopez-Brea M. [High percentage of clarithromycin and metronidazole resistance in Helicobacter pylori clinical isolates obtained from Spanish children]. Rev EspQuimioter 2009;22:88-92.

25. Heo J, Jeon SW, Jung JT, Kwon JG, Lee DW, Kim HS, et al. Concomitant and hybrid therapy for Helicobacter pylori infection: A randomized clinical trial. J Gastroenterol Hepatol 2015;30:1361-6. doi: 10.1111/jgh.12983.

26. He L, Deng T, Luo H. Meta-analysis of sequential, concomitant and hybrid therapy for Helicobacter pylori eradication. Intern Med 2015;54:703-10. doi: 10.2169/ internalmedicine.54.3442.

27. Talebi Bezmin Abadi A, Mobarez AM, Taghvaei T, Wolfram L. Antibiotic resistance of Helicobacter pylori in Mazandaran, North of Iran. Helicobacter 2010;15:505-9. doi: 10.1111/j.1523-5378.2010.00795.x. 